\title{
An infant with isolated ventricular non-compaction: a familial cardiomyopathy
}

\author{
Manouri P Senanayake ${ }^{1}$, Sumudu Seneviratne ${ }^{2}$, Duminda Samarasinghe ${ }^{3}$ \\ Sri Lanka Journal of Child Health, 2011; 40: 76-77
}

(Key words: isolated ventricular non-compaction; IVNC; familial cardiomyopathy)

\section{Introduction}

Isolated ventricular non-compaction (IVNC) is an under diagnosed familial cardiomyopathy which is frequently misdiagnosed as dilated or hypertrophic cardiomyopathy ${ }^{1,2}$. Awareness of this condition is important because the outcome in infants is not invariably fatal. We report an infant of Sri Lankan parentage with isolated ventricular non-compaction whose older sibling had died during infancy with a diagnosis of 'dilated cardiomyopathy'.

\section{Case history}

An exclusively breast fed baby boy, who had caused concern due to poor postnatal weight gain of only $15 \mathrm{~g} /$ day, presented with heart failure at seven weeks of age. His birth weight at term was $2.1 \mathrm{~kg}$. On examination, he looked wasted and the weight was well below the 3 rd centile. Length and occipitofrontal circumference were between the 3rd and 10th centiles. He looked unwell, was tachypnoeic and had dysmorphic features such as prominent eyes, triangular face and receding chin. There was no cyanosis or oedema. Heart rate was 140 beats per minute and a grade 2 systolic murmur was audible at the left sternal edge. Breathing was vesicular with no added sounds; liver was soft and palpable $2 \mathrm{~cm}$ below right costal margin.

Gross cardiomegaly was seen on the chest $\mathrm{x}$ ray and electrocardiography showed normal sinus rhythm and left axis deviation with no evidence of ventricular hypertrophy or bundle branch block. On echocardiography there were two well formed ventricles and dilated cardiac chambers with non compaction of the left venticular myocardium. Left ventricular ejection fraction was $20 \%$.

\section{${ }^{1}$ Professor in Paediatrics, Faculty of Medicine, Colombo, ${ }^{2}$ Registrar, Lady Ridgeway Hospital for Children Colombo, ${ }^{3}$ Consultant Paediatric Cardiologist, Lady Ridgeway Hospital for Children Colombo}

(Received 4 August 2010: Accepted 24 September 2010)
He was commenced on digoxin, frusemide, spironolactone and captopril and the heart failure resolved. When reviewed one month later he was thriving along his birth weight centile and apart from the soft murmur, there were no cardiac abnormalities detected clinically. Implications for future pregnancies were discussed with the parents.

He was the youngest of three boys born to nonconsanguineous parents. An older sibling who had 'dilated cardiomyopathy with profound systolic dysfunction' died aged six months and an autopsy was refused by the parents. The surviving sibling appeared healthy at 4 years.

During the second pregnancy the mother had developed chest pain and mild apical hypokinesia was noted on echocardiography. Subsequent echocardiography showed a normal ejection fraction. Screening echocardiography and electrocardiography were normal on the father and sibling.

\section{Discussion}

Myocardial compaction is a normal structural change that occurs between five to eight weeks of fetal life. An arrest of this normal process constitutes the entity known as non-compaction of the myocardium which is characterized by persistence of prominent ventricular trabeculations and deep inter-trabecular recesses. It typically involves the apical or middle portion of left ventricle, although involvement of right ventricle has been reported ${ }^{1,2}$.

Diagnosis of IVNC in our patient was based on characteristic echocardiographic findings of multiple trabeculations, deep intertrabecular recesses communicating with the ventricular cavity and a two layered endocardium with a non-compacted to compacted ratio greater than 2 .

Although mortality is high due to heart failure, thrombo-embolic events or ventricular arrhythmias, an "undulating phenotype" has also been described with a recovery period of variable length ${ }^{2}$. 
It is believed that IVNC accounts for almost $10 \%$ of cardiomyopathies $^{2}$. A familial incidence with Xlinked recessive, autosomal recessive and autosomal dominant inheritance patterns are reported ${ }^{1,4}$. Our finding of a previous diagnosis of "dilated cardiomyopathy" in a deceased sibling indicates the need for greater awareness of this distinct cardiomyopathy.

\section{References}

1. Jenni R, Oechslin E, Schneider J. Echocardiographic and patho-anatomical characteristics of isolated left ventricular noncompaction: a step towards classification as a distinct cardiomyopathy. Heart 2001; 86: 66671.

2. Pignatelli RH, McMahon CJ, Dreyer WJ, Denfield SW, Price $J$ et al. Clinical characterization of left ventricular noncompaction in children: A relatively common form of cardiomyopathy. Circulation 2003; 108: 2672-8.

3. Lorsheyd A, Cramer MM, Velthuis BK, Vonken EP, Smagt J et al. Familial occurrence of isolated non-compaction cardiomyopathy. European Journal of Heart Failure 2006; 8: 826 -31.

4. Ichida F, Hamamich Y, Miyawaki T, Ono Y, Kamiya T. Clinical features of isolated noncompaction of the ventricular myocardium: Long-term clinical course, hemodynamic properties and genetic background. Journal of the American College of Cardiology 1999; 34: 233-40. 